\title{
Immune Responses in Mice Vaccinated with Virus-Like Particles of Western Equine Encephalitis Virus from an Insect Cell-Based Expression System
}

\author{
Jinzhu Ma $\mathrm{Ma}^{1}$, Hualei Wang ${ }^{1}$, Xuexing Zheng ${ }^{1}$, Hongxia Wu $\mathrm{Wu}^{2}$, Songtao Yang $^{1}$, and \\ XIanzhu Xia ${ }^{1}$ \\ ${ }^{1}$ Academy of Military Medical Science of Chinese People's Liberation Army \\ ${ }^{2}$ Academy of Military Medical Science of PLA
}

January 8, 2021

\begin{abstract}
Western equine encephalitis virus (WEEV) can cause lethal encephalitis in humans and equines and represents a serious public health threat in many countries. Therefore, development of efficient vaccines against WEEV remains an important challenge in the field of disease control. This study described for the first time successful production of WEEV virus-like particles (VLPs) in insect cells using recombinant baculoviruses. This well-established expression system is very suitable for production of WEEV VLPs. The immune experiment herein in mice showed that the VLPs formulated with 206-adjuvant were responsible for the stronger-VLP-specific cellular immune response, and were able to induce the secretion of IL-2, IL-4, IFN- $\gamma$ and production of high titer antibodies that can effectively neutralize the WEEV pseudoviruses. The WEEV VLPs from insect cells could provide a new, safe, non-replicating and effective vaccine candidate against WEEV infections.
\end{abstract}

\section{Hosted file}

Jinzhu ma-Research paper.pdf available at https://authorea.com/users/388137/articles/502977immune-responses-in-mice-vaccinated-with-virus-like-particles-of-western-equine-

encephalitis-virus-from-an-insect-cell-based-expression-system

\section{Hosted file}

Jinzhu ma-figure-.pdf available at https://authorea.com/users/388137/articles/502977-immuneresponses-in-mice-vaccinated-with-virus-like-particles-of-western-equine-encephalitisvirus-from-an-insect-cell-based-expression-system 